\title{
SUR L'UTILISATION DES ANTIBIOTIQUES EN PISCICULTURE
}

\author{
Christian MICHEL \\ Docteur Vètérinaire \\ Laboratoire d'ichtyopathologie, I.N.R.A. \\ 78850 THIVERVAL - GRIGNON
}

\section{DEFINITION}

Les antibiotiques sont des substances antimicrobiennes d'origine biologique, qui agissent à faible concentration sur les microorganismes en bloquant certaines étapes métaboliques indispensables à leur survie ou à leur croissance.

Un tel mode d'action ne se traduit pas forcément par la mort de l'agent infectieux, mais sa croissance est toujours interrompue. Dans ce cas, l'organisme a le temps d'édifier sa réponse et ce sont les mécanismes de défense qui en dernier ressort éliminent le germe.

\section{CLASSIFICATION}

Les antibiotiques ont été regroupés en familles dont la distinction repose sur la structure chimique et les modes d'action. Ces familles, qui actuellement sont au nombre de 8 auxquelles il faut ajouter les sulfamides, groupent des substances dont les mécanismes d'action et le spectre d'activité sont identiques. Ainsi, les $\beta$ lactamines, qui comprennent la pénicilline et les antibiotiques apparentés, agissent toutes au niveau de la paroi, et seulement chez certaines espèces bactériennes.

D'autres familles inhibent les synthèses protéiques, d'autres la régulation des transferts d'eau et d'électrolytes. Les Sulfamides sont des analogues de structure d'un métabolite intermédiaire auquel ils se substituent dans une réaction biochimique.

Article available at http://www.kmae-journal.org or http://dx.doi.org/10.1051/kmae:1978012 


\section{RESISTANCES}

Il existe des germes naturellement réfractaires à l'action de certains antibiotiques. D'autres, initialement sensibles, ont acquis une résistance à l'endroit de certaines molécules. Ces résistances ont un support génétique, héréditaire, et posent de graves problèmes pour l'antibiothérapie.

\section{PRINCIPES GENERAUX DE L'ANTIBIOTHERAPIE}

L'antibiothérapie a pour objet la destruction des microorganismes pathogènes par apport d'une concentration efficace d'antibiotique au site de prolifération des germes. Les traitements par antibiotiques doivent obéir à certains impératifs:

- Le choix de l'antibiotique est fonction de la sensibilité du germe en cause. La réalisation d'un antibiogramme permet de connaitre les différentes sensibilités et de fixer ce choix en toute certitude.

- L'antibiotique doit pénétrer dans la cellule bactérienne pour exercer son activité et par conséquent être présent en quantité suffisante au niveau du foyer bactérien visé. II est possible de jouer sur la dose, dans les limites compatibles avec une absence de toxicité pour le sujet traité. La voie d'administration sera choisie en fonction du site à atteindre. Pour ce faire, il est important de connaitre le devenir de la substance dans l'organisme.

- L'action doit être prolongée. Contrairement aux agents antiseptiques. l'antibiotique ne crée pas de désordre immédiat et irréversible dans la cellule microbienne. Il faut donc maintenir sa présence jusqu'à ce que l'interruption de la fonction vitale conduise la cellule à la destruction spon. tanée ou à l'élimination par l'organisme.

Le non respect de ces règles expose non seulement à l'échec, mais au risque de voir apparaitre des résistances qui compliquent d'autant les interventions ultérieures.

\section{INDICATIONS EN PISCICULTURE}

L'antibiothérapie est une arme thérapeutique. Tout emploi préventif est à proscrire. Ceci découle des règles d'utilisation énoncées. De plus, l'eau est un milieu écologique fragile, où toute rupture d'équilibre peut avoir des conséquences néfastes. [On sait que certaines maladies traduisent une perturbation du milieu aquatique (myxobactérioses par exemple)]. II est dangereux d'intervenir sans y être contraint.

La suspicion de maladie bactérienne est affaire d'expérience. Dès les premières mortalités suggérant une telle origine, un antibiotique à large spectre (tétracycline) pourra être mis en œuvre. On prendra garde à maintenir un faible effectif d'animaux non traités, qui pourront le cas échéant être envoyès au laboratoire de diagnostic. Les résultats de l'antibiogramme permettront alors la correction du tir. Les maladies justiciables de cette attitude sont la Furonculose, les infections à Aeromonas et Pseudomonas (hydropsie infectieuse, pourritures des nageoires et de la queue), l'Erythrodermatite des carpes, les Myxobactérioses branchiales et cutanées (pour lesquelles le Furanace donne d'excellents résultats). 


\section{ANTIBIOTIQUES UTILISES}

Certains antibiotiques ne s'administrent que par injection parentérale et sont donc peu intéressants pour la pisciculture. Le prix de revient du traitement est également un facteur limitant. Enfin le bon sens suggère, face à la multiplication des résistances, de renoncer à l'emploi de certaines molécules très précieuses pour la médecine, dont l'utilisation aveugle en élevage, où l'on ne dispose pas toujours de l'antibiogramme en temps utile, est une pratique imprudente.

En usage courant, on se limitera à l'emploi de substances à large spectre d'activité : tétracyclines, sulfamides. Les furanes (Furanace) seront utiles pour les infections branchiales. Exceptionnellement, après réalisation de l'antibiogramme, on pourra recourir au chloramphenicol, à l'ampicilline, à la colistine et au trimethoprime. L'association de ce dernier avec la sulfamethoxazole (ND Tribrissen) est parfois très efficace contre la Furonculose.

Les associations d'antibiotiques sont peu utilisèes en pisciculture du fait de leur prix de revient. II y a pourtant intérèt, lorsque les circonstances s'y prêtent, à associer un traitement de désinfection externe, aux ammoniums quaternaires par exemple, qui ajoute ses effets à ceux de l'antibiotique (cas de lésions externes).

\section{MODE D'ADMINISTRATION}

La voie d'administration la plus pratique et la plus efficace est la voie orale, l'antibiotique étant incorporé à l'aliment. La balnéation est parfois employée ; mais son intérêt reste limitè à certains cas (faibles volumes d'eau à traiter, infections des tissus cutanés et des branchies).

\section{DUREE DU TRAITEMENT}

Le traitement doit être prolongé 6 jours au minimum. Il est conseillé d'opérer deux traitements à une semaine d'intervalle.

\section{POSOLOGIES}

Les doses employées seront déterminées par le vétérinaire, selon le type d'infection, le site à atteindre au niveau du poisson et le résultat de l'antibiogramme.

En conclusion, les antibiotiques représentent une arme de choix contre les infections bactériennes. Leur emploi exige cependant le respect de règles simples, mais strictes. C'est pourquoi on ne l'envisagera qu'après consultation d'une personne habilitée à en faire la prescription. 